\title{
CONSTRUCTION SCHEDULE SIMULATION FOR IMPROVED PROJECT PLANNING: ACTIVITY CRITICALITY INDEX ASSESSMENT
}

\author{
Pei Tang \\ Project Controls Engineer, \\ Jois Construction Management System, Inc. \\ 1741 Whitehorse Mercerville Rd, \\ Mercerville, NJ 08619, USA. \\ Amlan Mukherjee \\ Associate Professor, \\ Department of Civil \& Environmental Engineering, \\ Michigan Technological University, \\ Houghton, MI 49931, USA.
}

\author{
Nilufer Onder \\ Associate Professor, \\ Department of Computer Science, \\ Michigan Technological University, Houghton, MI 49931, USA.
}

\begin{abstract}
The objective of this paper is to illustrate the application of construction schedule simulation during the planning phase of a construction project. Schedule simulations can be used to develop risk informed schedules that support improved contingency planning. This paper discusses the challenges underlying construction schedule simulation, and how they can be addressed by an interactive simulation platform such as the Interactive Construction Decision-Making Aid (ICDMA). Further, the paper uses ICDMA during the planning process, for a case study, to identify the activities in a project that are most likely to change criticality during the construction process. Current scheduling techniques such as the Critical Path Method (CPM) are capable of identifying critical activities, but they cannot be used to assess the likelihood of alternative critical paths emerging during construction due to unexpected disruptions in the as-planned schedule. The research illustrates the usefulness of construction simulation in supporting the scheduling process.
\end{abstract}

\section{INTRODUCTION}

Dealing with uncertainty in construction management presents a dynamic decision-making problem. External events such as inclement weather, labor disturbances, or unexpected rework can impact the expected outcome of a project profoundly. The specific time in the schedule at which such events occur also significantly influences the extent of the impact. In addition, such events have cascading impacts on the schedule that may reinforce existing delays, and/or set in motion unexpected feedback. Thus, all or as many possible deviations from the as-planned schedule must be considered carefully at the planning phase.

Consider the following example: during project planning, the Critical Path Method (CPM) is the most widely used project scheduling tool. However, it does not account for deviations in the project schedule due to uncertain events. During construction, activities are interrupted due to impacts of uncertain events like severe weather, and other unforeseen events that result in delaying activities and altering the critical path. Often such delays are outcomes of requests for information that can impact resource constraints without directly impacting the critical path and in turn cause unrecoverable delays. From a project management 
perspective, this means that given uncertain conditions, non-critical activities may have a likelihood of becoming critical during the project execution. Advance knowledge of the likelihood of activities to become critical can help in identifying appropriate contingencies and best management strategies. Currently there are few methods to develop such risk informed schedules.

Hence, the objective of this research is to develop simulation based methods that can be used to understand how a specific schedule behaves under uncertainty for a given question. Specifically, the paper presents a case study using the Interactive Construction Decision-Making Aid (ICDMA), simulation platform to identify a criticality index for each activity, for a given set of uncertain conditions. This index is referred to as the Activity Criticality Index (ACI) and is defined as the probability of an activity becoming critical. The paper presents a general method to predict an activitys ACI during the construction process given a construction decision-making strategy.

\section{SCHEDULE SIMULATION}

Construction schedule simulations are fundamentally different from simulations of construction operations. The nature of construction operations makes them suitable to modeled as activity cycle diagrams (ACD) using discrete events. Uncertainty in parameters (such as truck loading times) can be observed through direct site data collection and relevant underlying distributions identified. Such information can be used to model, simulate and estimate critical factors such as the duration of operations. Various research efforts have successfully modeled similar and more complicated questions, and their relevance to this research has been discussed in previous publications (Rojas and Mukherjee 2005, Rojas and Mukherjee 2006).

Simulating a construction schedule presents a different set of challenges. First and foremost, construction projects are non-prototypical, i.e., a project is built only once - and while each project provides lessons, unique project conditions often make it difficult to transfer knowledge between projects. Hence, simulations are the most suitable tool for planning such contingencies for such projects. Second, while projects do tend to have inherent structure (e.g. due to the linear or repetitive nature of work to be performed), ACDs and discrete events are not always the most suitable abstraction to model project information. If, the primary motivation of modeling schedules, is to understand their sensitivity to disruption, then a suitable abstraction must be able to represent and simulate a variety of disruptive events at any time during the duration of the project. This implies that at any and all time points there should be a mechanism to disrupt the schedule and let the cascading impacts be reflected through out the schedule.

Taken together the above challenges imply that a schedule simulation demands an abstraction in which project uncertainty is considered to be a function of the many possible futures that a project can traverse. This is a departure from representing uncertainty by characterizing variables with statistical distributions. Hence, given a distribution of a set of disruptive events, when and if any of them occur will dictate the future that the project traverses. Needless to say, we have access to only one single project future - the "as-built" schedule, while the "as-planned" schedule is the desired future. The non-prototypical nature of construction projects makes simulation validation particularly difficult.

The above challenges can be best addressed by representing construction projects using a continuous time advance platform. It should also be interactive in nature so that decision-makers can identify alternative strategies and use the simulation to test the response of a given schedule to different management strategies. The next section introduces the ICDMA, the simulation platform.

\section{ICDMA}

ICDMA is a continuous time advance simulation. It provides a rich state based representation of construction information and constraints that drives the construction management domain using system variables, random variables and decision variables. The underlying temporal representation differentiates it from traditional discrete event simulations - instead of advancing the simulated time from one discrete event to the next, it advances time from one time point to the next contiguous time point. The simulation is not dictated 
by a calendar of specific events, but instead it is an emulation of a passage through time. Hence, at each time point $\mathrm{T}$ the simulation is ascribed a state ST which is expressed by a constraint network of activities and events called the TONAE (Anderson, Mukherjee, and Onder 2009). The querying algorithm, which is a part of ICDMA, is used to develop risk distributions at the end of each time point. It queries the combinatorial future space of project outcomes using a Monte-Carlo sampling method at the end of each time point and classifies the results by impact and probability.

At the end of each time point $T$ (this could represent a months or years depending on the granularity) the simulation transitions to its new state $S_{T+1}$ at time point $T+1$ based on inputs (decisions and deliberations) made by a planner/decision-maker who has the ability to directly control the work flow in the simulated project. This implies that each time point in the simulation is effectively a control point allowing decisionmakers to experiment with different strategies and examine outcomes associated with alternative decisions. The simulation is capable of formally representing decisions at each time point using a set of decision variables - $\mathbf{C}_{T}$. Hence the rate of change of the system can be expressed mathematically by Equation (1):

$$
\mathbf{S}_{T+1}=\left[\mathbf{C}_{T}+\mathbf{R}\right] \bullet \mathbf{S}_{T}
$$

Where $\mathbf{R}$ is the impact of random events - such as bad weather, change orders, create on the progress of the project simulation. The $\bullet$ operation symbolizes the impact of the decisions and random variables on the current state of the system $\left(\mathbf{S}_{T}\right)$ to create the new state of the system $\mathbf{S}_{T+1}$. Currently the simulation can be used to represent the impact of external events and decisions on the evolution of the simulation from state to state. Unlimited control points in the simulation also provides an interactive platform capable of providing project specific, context rich strategy analysis and contingency plans. Alternative project management strategies can be compared and their impacts on eventual project outcome can be studied in the simulated project environment by statistically analyzing this stored simulation data to infer and compare the relationships symbolized by the $\bullet$ operation for each strategy.

In this paper we introduce the use of ICDMA as an experimental test bed for evaluating alternative strategies and supporting the iterative decision-making process. Figure 1, illustrates the conceptual layout of the integrated system. The dotted line separates the project managers area of involvement from the simulation developers. While the latter enables the platform, the former uses it to interactively experiment with alternative strategies: iteratively generating new strategies and experimenting with them to refine the final set of management strategies. This iterative process supports decision-making process. The decisionmakers define the project or scenario to be studied and through a web-based interface that populates a back-end database with project specific information regarding constraints, event probabilities and conditions triggering events and existing after their occurrence. This information is used by the emulator and the simulator to automatically generate the TONAE and the deploy the simulation.

The simulation is run multiple times allowing interactive testing of alternative decision-making strategies. The emulator allows exploration of the project on a day to day basis. The simulator generates external disruptive events such as specified by the decision-maker. The emulator also updates the state of the simulated project at the end of each simulated time step and cascades the impacts of each disruptive event and subsequent decisions through the TONAE. ICDMA also provides feedback to the decision-makers regarding the as-planned and as-built states of the project. The following data from multiple simulation runs are archived in a back-end database:

- State information at the end of each time point $\left(\mathbf{S}_{T}, \mathbf{S}_{T+1} \ldots\right)$

- The list of external events $(\mathbf{R})$ and the times in the project when they occur

- All decisions made by the decision-maker in terms of resource allocated and schedule changes $\left(\mathbf{C}_{T}\right)$

- Outcomes from the querying algorithm at the end of each time point $T$.

The data is analyzed through statistical clustering methods to provide decision-makers the ability to compare and contrast the outcomes of alternative strategies. This information loops back into the strategy generation phase allowing them to generate and test hybrid strategies. Such a platform is critical to planing and 


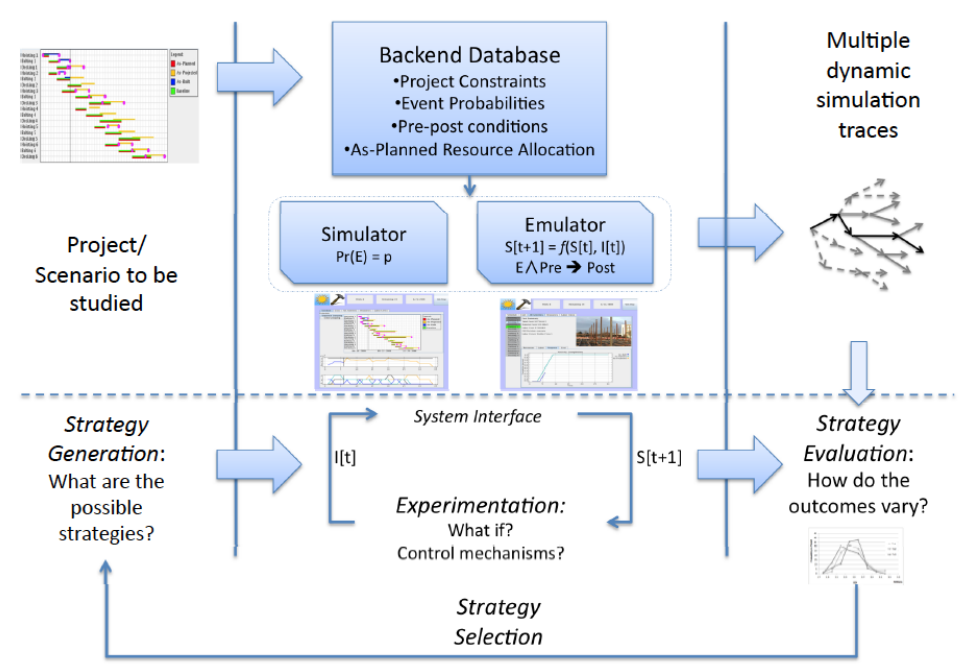

Figure 1: Conceptual Description of Platform.

decision-making for complex construction projects in which the occurrence of the same external event say a bad weather event - at different times in the schedule can have very different project outcomes.

\section{ACTIVITY CRITICALITY INDEX}

The construction industry has difficulties with using Critical Path Method (CPM) as a project control tool. Traditionally, an activity is identified as either critical or non-critical only at the planning stage, which has limited the ability of CPM tools to control a project after it starts. After a project starts, activity progress may be interrupted by uncertain events that change the constraint relationships between the activities. The changes sometimes force non-critical activities to become critical and vice versa. In addition, the specific time in the schedule when the critical path changes can also significantly impact formulation of resource allocation plans. The paper presents a general method to predict an activitys ACI during the construction process given a construction decision-making strategy.

A decision-making strategy is defined as a guideline, that provides a family of acceptable resource allocation decisions in order to achieve project goals (schedule, budget) while accounting for impacts of uncertain events. Identifying the ACI of an activity can help construction managers formulate resource allocation plans, and improve their construction management strategies. Methodologically, the significance of this research is that it applies the CPS technique within a dynamic simulation environment and presents a general method to support resource allocation decisions before the project starts.

\subsection{Background}

The background literature discusses various methods that are relevant to calculating the ACI of an activity. One approach is to examine historical construction decision data sets. Soibelman and Kim (2002) and Kim, Soibelman, and Grobler (2008) use Knowledge Discovery in Databases (KDD), a data analysis process, to identify main factors of construction delays using statistical measurements and a decision tree based machine learning algorithm. Other methods like regression models (Lucko and Mitchell 2010), pattern analysis (Battikha 2008), and automatic hierarchical classification method (Caldas and Soibelman 2003) have also been used to mine construction project documents with the goal of identifying improved management strategies. 
However, such methods allow only a partial observability of the success or failure of a decision. Instead, this research adopts an interactive simulation method to examine the complete project outcomes given a construction management strategy. The interactive simulation platform used is called Interactive Construction Decision Making Aid (ICDMA), a continuous time advance simulator that simulates CPM scheduled construction projects. It allows decision makers to respond to project contingencies by (re)allocating resources during the simulation process to explore what-if scenarios and multiple deviations from the as-planned project progress (Tang, Mukherjee, and Onder 2010a, Tang, Mukherjee, and Onder 2010b).

Therefore, for this research, the point of departure from the existing body of research is that it uses a simulation based approach to explore alternative project realizations, and deviations from the intended critical path. Similar to previous efforts, the proposed method is based on an analysis of a completed construction project schedule and documents. However, instead of statistically analyzing schedule performance across multiple projects that may or may not have shared similar circumstances, the proposed method statistically analyzes multiple alternative realizations of a single project within an uniformly simulated environment. The likely outcome of the proposed method is to provide deeper insight on a project-by-project basis thus enhancing project planning approaches.

\section{METHODOLOGY}

The proposed methodology applies the Critical Path Segment (CPS) technique (Hegazy and Menesi 2010) for representing schedule information, within an interactive simulation environment, ICDMA, to experimentally explore possible deviations from the as-planned critical path. The motivation for using the CPS method is that it allows the representation of an activity at a finer level of granularity. Different from traditional representation of an activity as a solid bar that spans a given duration, the CPS technique decomposes the duration of each activity into a number of separate, but connected time segments that add up to the total duration of the activity. For example, if the scheduled duration for paving concrete is 30 days, 30 time segments are used to represent it. Hence, this representation makes it possible to represent any logical relationship (finish-to-start, finish-to-finish, start-to-finish, and finish-to-finish) and lags by only finish-tostart relationship. It also allows scheduling activities with not only time-based, but also production-based methods. When the CPS technique is used in conjunction with ICDMA, it allows for a closer examination of each activity segment's criticality by performing multiple simulation trials. The as-planned and as-built schedules from a real life highway construction project are used so that the simulation results can be checked for validity against the actual project outcome.

The general method presented in this paper consists of the following steps:

1. Identify project constraints and uncertainty information from the construction project being studied. Sometimes the constraints may be in space and/or driven by safety requirements. All such constraints are converted to equivalent temporal constraints.

2. Develop time-based and production-based as-planned schedules using the CPS technique.

3. Set up project simulation environment in ICDMA using the as-planned schedules and project constraints identified in the previous steps.

4. Design the simulation experiment using ICDMA and conduct simulation experiments for alternative decision-making strategies.

5. Analyze simulation outcomes and calculate the ACI for each activity segment.

The rest of the paper discusses each of the above steps in the context of a case study project.

\section{IMPLEMENTATION}

The case study is a ten-mile concrete pavement re-construction project in southeast Michigan. This research studied the reconstruction of the east bound section. The east bound section consisted of fourteen major activities (Table 1). The activities and durations were obtained from the as-planned schedule and the progress 
Table 1: Activity Duration and Technical Precedence.

\begin{tabular}{|l|l|l|}
\hline Activity & Duration & $\begin{array}{l}\text { Precedence } \\
\text { activities }\end{array}$ \\
\hline 1_Strip topsoil & 10 days & N/A \\
\hline 2_Remove concrete pavement & 30 days & 1 \\
\hline 3_Grade subbase & 26 days & 2 \\
\hline 4_Install drainage & 18 days & 2,3 \\
\hline 5_Place open-graded drainage course (OGDC) mainline & 18 days & $2,3,4$ \\
\hline 6_Pave east bound (E.B.) mainline & 32 days & 5 \\
\hline 7_Place OGDC ramps and gaps & 8 days & $4,5,6$ \\
\hline 8_Pave E.B. gaps and ramps & 9 days & 7 \\
\hline 9_Place gravel shoulder & 4 days & 6,8 \\
\hline 10_Slope grading and restoration E.B. & 26 days & 8 \\
\hline 11_Stripe to open pavement E.B. & 3 days & 10 \\
\hline 12_Relocate barrier wall & 10 days & 11 \\
\hline 13_Re-stripe west bound & 3 days & 12 \\
\hline 14_All lanes open & 1 days & $9,10,13$ \\
\hline
\end{tabular}

schedule (MDOT Form 1130) provided by the contractor. The project was scheduled to be completed in 106 working days and was actually completed in 129 working days.

\subsection{Step 1: Activity Constraints and Uncertainty Information}

In a construction project, activities are related by constraint relationships. Besides the precedence determined by technical constraints, safety constraints between the processes should be considered as well. The safety distances are determined on the assumption that two activities cannot occupy the same working spot. For two activities $i$ and $j$ (assuming that Activity $i$ precedes Activity $j$ ), the minimum distance between them at the beginning of Activity $i$ is defined in Equation (2):

$$
D_{i j} \geq \max \left\{t * \frac{L}{T_{j}},\left(1-\frac{T_{i}}{T_{j}}\right) * L\right\}
$$

where $D_{i j}$ represents distance between Activity $i$ and Activity $j, L$ represents the length of the project, $t$ is the buffer time between the activities, and $T_{i}$ and $T_{j}$ represent the duration of Activities i and $\mathrm{j}$ respectively. Buffer time is subject to technical constraint and is determined by the schedulers' experience. As CPM uses temporal relationships to relate activities, the minimum distances between them are converted into temporal constraints. For two activities $i$ and $j$ (assuming that Activity $i$ precedes Activity $j$ ), if the safety distance is $D_{i j}$, the time that Activity $j$ should start later than Activity $i$ is determined by $T_{i j} \geq \frac{1}{T_{i}} * D_{i j}$, where $T_{i j}$ represents the temporal constraint that is equivalent to distance constraint $D_{i j}$.

For this case, the distance constraints identified by the contractor were: one mile between Activities 1 and 2, two miles between Activities 2 and 3, three miles between Activities 3 and 4, four miles between Activities 4 and 5, six miles between Activities 5 and 6, seven miles between Activities 6 and 7, three miles between Activities 8 and 9, and one mile between Activities 10 and 11. Using the distance and temporal constraint conversion equation, the temporal constraints between activities were: (1) Activity 2 started one day after the beginning of Activity 1, (2) Activity 3 started six days after the beginning of Activity 2, (3) Activity 4 started eight days after the beginning of Activity 3, (4) Activity 5 started six days after the beginning of Activity 4, (5) Activity 6 started eleven days after the beginning of Activity 5, (6) Activity 7 started twenty-five days after the beginning of Activity 6 and zero days after completion of Activity 5, (7) Activity 8 started zero days after the completion of Activity 7, (8) Activity 9 started three days after the beginning of Activity 8 and zero days after the completion of Activity 6, (9) Activity 10 started zero days 
after the completion of activity 8, (10) Activity 11 started two days after the beginning of activity 10, (11) Activity 12 started zero days after the completion of Activity 11, (12) Activity 13 started zero days after the completion of Activity 12, and (13) Activity 14 started zero days after the completion of Activity 9, Activity 13 and Activity 10.

During the construction, uncertain events usually occur and interrupt the project activities. To observe the changes in critical paths, the following event-consequence-probability tuples were considered: (1) bad weather, reducing productivity to half on all activities, occurrence probability of $20 \%$, (2) equipment failure or worker sickness, occurrence probability of $12 \%$, (3) concrete testing failure, resulting in stoppage of paving east bound mainline or paving east bound gaps and ramps for one day, occurrence probability of 5\%, and (4) high water table, causing stoppage on grading subbase for one day waiting to change new subbase material, occurrence probability of $10 \%$. The events, consequences and probabilities were obtained from directly mining the as-built project records and documents that were directly collected with the help of project site inspectors (Tang, Cass, and Mukherjee 2013).

\subsection{Step 2: Develop CPS Time-based and Production-based Scheduling}

Different from traditional CPM scheduling, the CPS technique uses only finish-to-start relationship to represent all constraint relationships between activities. The activities, associated durations, and constraints between them were used to create critical path diagrams. Figure 2 shows the critical path diagram using time-based scheduling method. The constraints were set only between the beginning of each activity. For example, Activity 3 was scheduled to start six days after Activity 2 began. Therefore, the first segment of Activity 3 started after the completion of the first segment of Activity 2, whose duration was six days. As a result, the total duration was 101 days and the critical activities were identified in dark color. For production-based scheduling method (Figure 3), the constraints were set between the segments of activities. In addition to the first segment of Activity 3 starting after the completion of the first segment of Activity 2, constraints were also set between the end of the second, third, fourth, and sixth segments of Activity 2 and the beginning of the second, fourth, fifth, and seventh segments of Activity 3 . The scheduled duration was 111 days, which was 10 days longer than scheduled with time-based method. In addition, the critical activities identified in two methods were different.

\subsection{Step 3: ICDMA Simulation Setup}

ICDMA simulates a construction project based on its resource-loaded as-planned (RLAP) schedule and its project environment. The RLAP schedule of the highway reconstruction project consisted of: (1) activities to complete the project and the estimated durations; (2) material, labor, and equipment usages for each activity; (3) unit price of labor, equipment, and material along with unit space occupied by each material; (4) as-planned schedule (Figures 2 and 3). The activity information is summarized in Table 1. The process of obtaining the second and third data sets can be found in co-authors' previous work (Tang, Cass, and Mukherjee 2013). All the information was input into a database to complete the project set up process.

\subsection{Step 4: Experimental Design}

After the set up of simulated project, experiments were conducted in a situation where the uncertain events were turned off to verify the simulation results. The simulated as-built results from time-based schedule and production-based schedule were exactly the same as scheduled, which verified that the simulation platform produced the correct intended results.

As it assumes that criticality alternations are stable given a construction management strategy, two decision strategies were designed. Control Strategy manages the schedule by taking the minimum number of actions in dealing with interruptions, which was implemented to compare the abilities of the time-based method and the production-based method in representing activity criticality. Catch Up Strategy, aiming at completing the project on time, was implemented on production-based schedule to estimate ACIs of each 


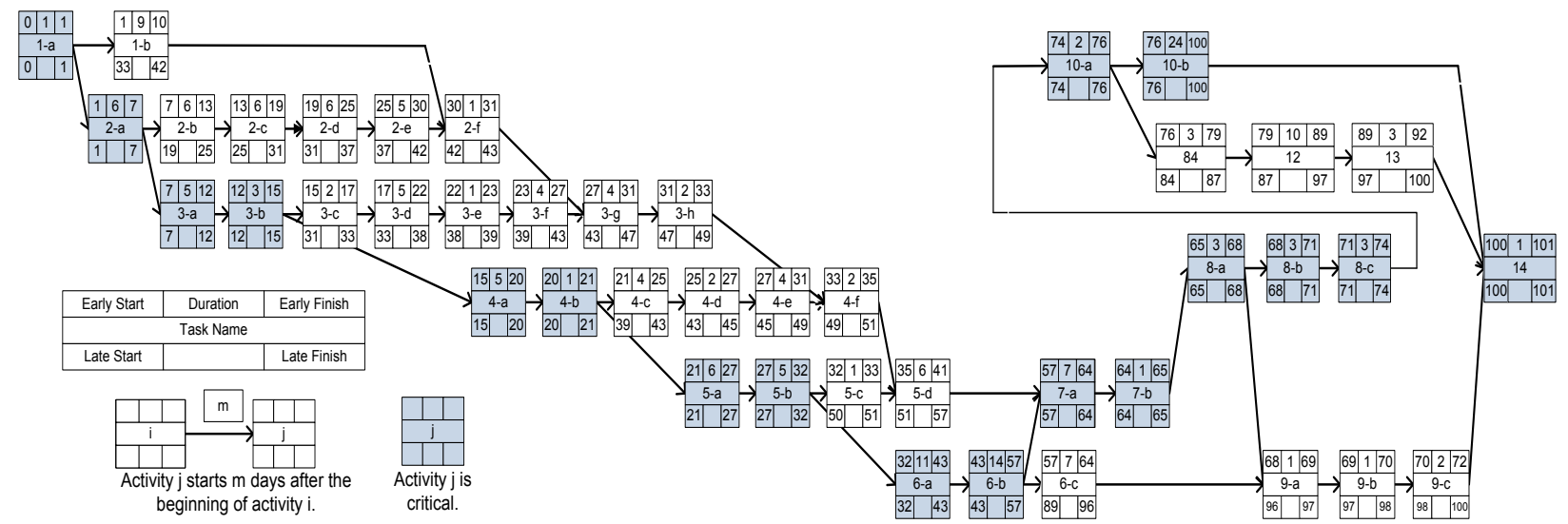

Figure 2: Time-based Critical Path Scheduling.

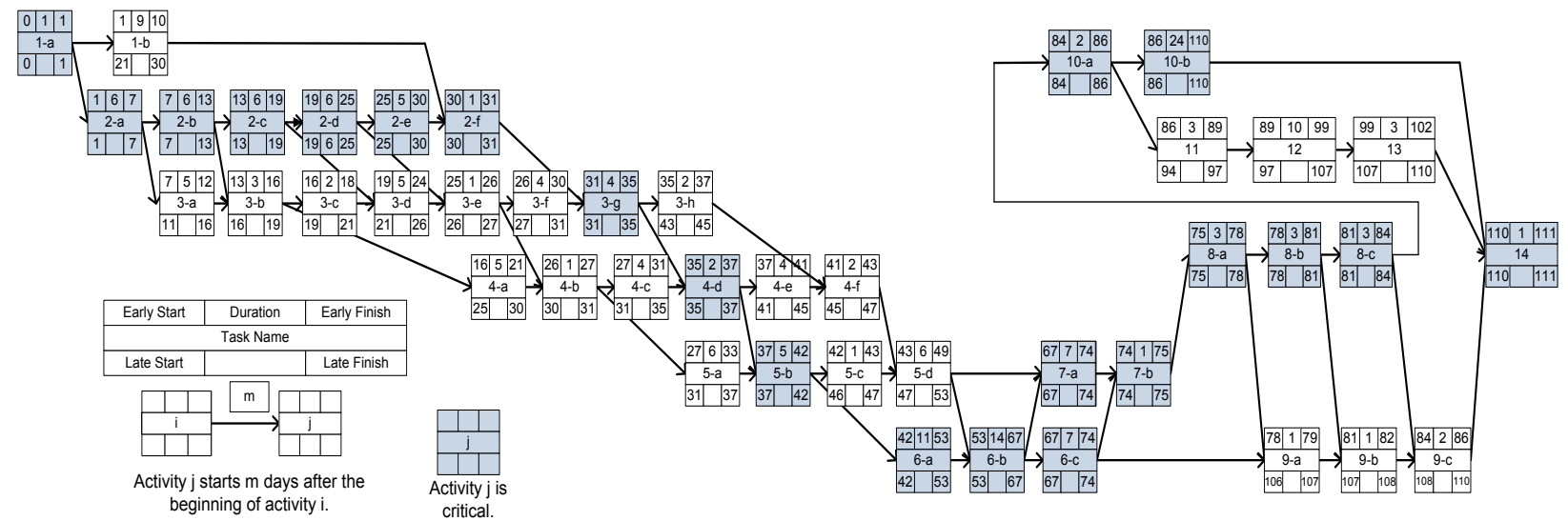

Figure 3: Production-based Critical Path Scheduling. 
activity. Details about the strategy definition and establishment can be found in authors' previous work (Tang, Mukherjee, and Onder 2010a, Tang, Mukherjee, and Onder 2010b). Each strategy was implemented for forty runs to meet the minimum requirement of statistical analysis. For the sake of uniformity, a single decision maker (the author in this case) ran all the simulations. The changes of critical paths were recorded for analysis.

- Control Strategy: It manages the schedule by taking the minimum number of actions in dealing with interruptions. The implementation is reflected by the following resource allocation policies: (a) labor crew policy: no extra workers are replaced in any case; (b) equipment policy: equipment is not fixed on the same day in any case; (c) space policy: space is allocated first to the critical activities; (d) no actions are taken when the project is falling behind.

- Catch Up Strategy: It manages the project schedule passively. The implementation is reflected by the following resource allocation policies: (a) labor policy: extra workers are hired and replaced in cases of illnesses; (b) equipment policy: equipment is fixed by the mechanics immediately; (c) space policy: space is allocated first to the critical activities; (d) actions are taken to catch up the schedule every time the project is three days behind the as-planned schedule.

\subsection{Step 5: Critical Path Analysis}

A total of 80 instances of the project realization with simulated as-built project process were collected when implementing Control Strategy to time-based and production-based schedules. Another 40 instances were collected when applying Crash Strategy to the production-based schedule. The average simulated as-built completion durations for three cases were $125.7,139.1$, and 112.6 working days, which were comparable to the as-built completion duration (129 working days).

Table 2 shows the average ACIs for each activity segment. ACI is defined in Equation (3):

$$
A C I=\frac{\text { Days being critical }}{\text { Simulated as-built duration }}
$$

The second and fourth columns shows the as-planned critical activities in Figures 2 and 3, represented by $\mathrm{X}$. The third and fifth columns have the ACIs (range from 0 to 1 ) for each activity. The higher the value of ACI, the higher the probability that the activity segment will be critical when performed. Table 2 shows that some of ACIs in the fifth column were between 0 and 1, indicating production-based scheduling method was more capable to capture activities' criticality changes.

Figure 4 summarizes the average ACIs for each activity segment when applying Crash Strategy. The $\mathrm{x}$-axis represents the activity progress and $\mathrm{y}$-axis represents probability of being critical (ACIs). For example, the first $10 \%$ of the activity of stripping topsoil had a $100 \%$ chance of being critical. It indicated that more emphasis should be placed on the first $10 \%$ of Activity 1 because any interruption in the first $10 \%$ of the activity will result in project delays. For Activity 3, identified critical segment (Figure 3) was 3-g (between $72 \%$ and $92 \%$ of the activity). However, simulation results showed that if applying Crash Strategy, there was still a chance to be critical for the segments of Activity 3 between $19 \%$ and $72 \%$. For Activities 9 and 11, there was no chance of being critical.

\section{CONCLUSION}

This paper illustrates how a schedule simulation platform such as ICDMA can be used to improve the construction scheduling process. By comparing the scheduled and simulated critical activities, the production-based scheduling method was proven to be more capable to capture the criticality changes than time-based scheduling method. Given a Catch Up Strategy, activities' criticality in a highway reconstruction project was calculated (Figure 4).

The significance of the work lies in connecting ACIs with the construction progress of each activity. Generally, the proposed simulation based method can enable construction managers test different construc- 

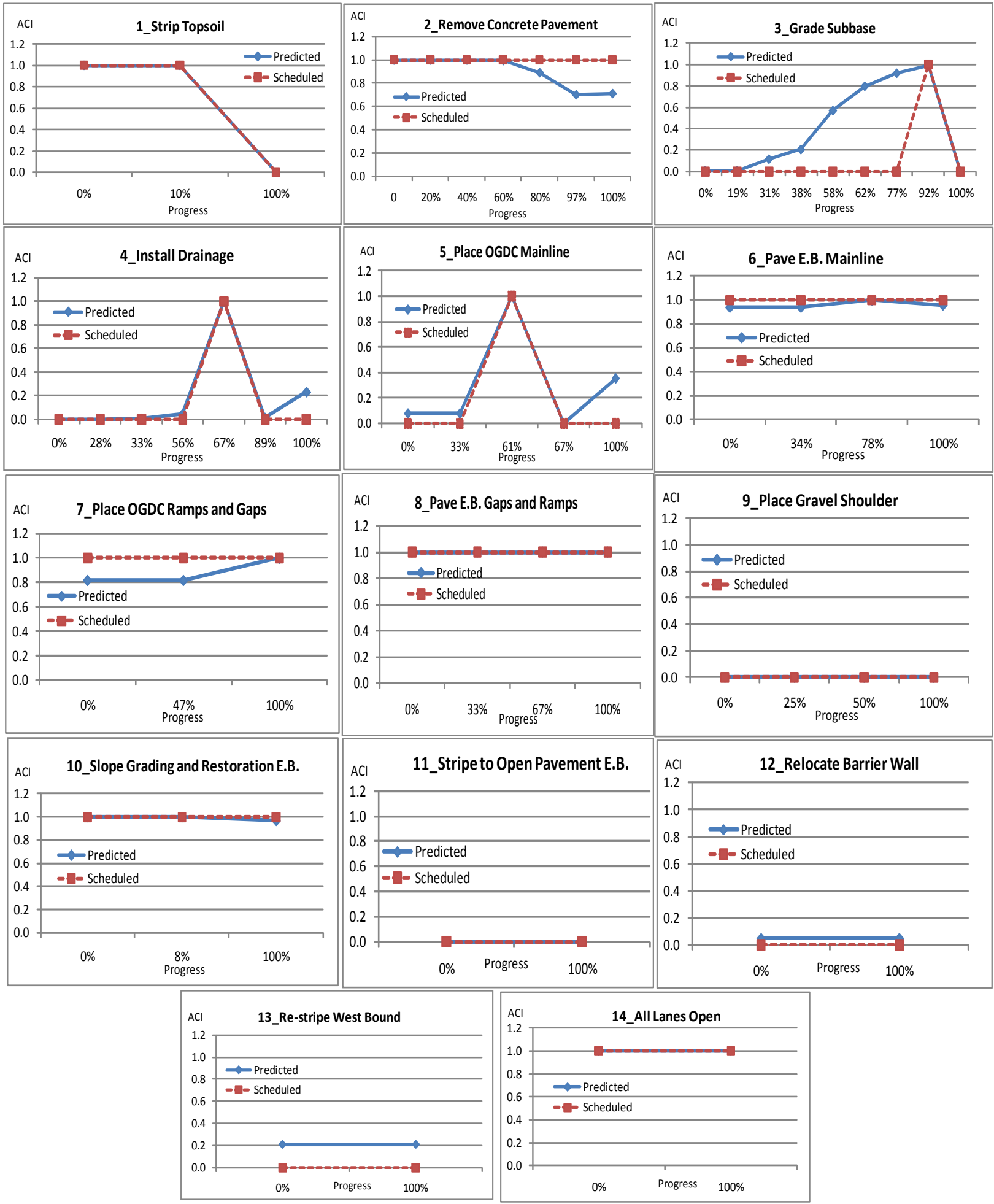

Figure 4: Activity Criticality Index (ACI) Prediction for Each Activity. 
Table 2: Scheduled and as-built Critical Activities.

\begin{tabular}{|c|c|c|c|c|}
\hline & \multicolumn{2}{|c|}{ Time-based } & \multicolumn{2}{|c|}{ Production-based } \\
\hline & Critical & $\mathrm{ACI}$ & Critical & $\mathrm{ACI}$ \\
\hline 1_Strip Topsoil(a) & $\mathrm{X}$ & 1.00 & $X$ & 1.00 \\
\hline 1_Strip Topsoil(b) & & 0.00 & & 0.00 \\
\hline 2_Remove Concrete Pavement(a) & $\mathrm{X}$ & 1.00 & $\mathrm{X}$ & 1.00 \\
\hline 2_Remove Concrete Pavement(b) & & 0.00 & $\mathrm{X}$ & 1.00 \\
\hline 2_Remove Concrete Pavement(c) & & 0.00 & $\mathrm{X}$ & 1.00 \\
\hline 2 Remove Concrete Pavement(d) & & 0.00 & $\mathrm{X}$ & 0.99 \\
\hline 2_Remove Concrete Pavement(e) & & 0.00 & $\mathrm{X}$ & 0.92 \\
\hline 2_Remove Concrete Pavement(f) & & 0.00 & $\mathrm{X}$ & 0.89 \\
\hline 3_Grade Subbase(a) & $\mathrm{X}$ & 1.00 & & 0.00 \\
\hline 3_Grade Subbase(b) & $\mathrm{X}$ & 1.00 & & 0.00 \\
\hline 3_Grade Subbase(c) & & 0.00 & & 0.00 \\
\hline 3_Grade Subbase(d) & & 0.00 & & 0.07 \\
\hline 3_Grade Subbase(e) & & 0.00 & & 0.23 \\
\hline 3_Grade Subbase(f) & & 0.00 & & 0.43 \\
\hline 3_Grade Subbase $(\mathrm{g})$ & & 0.00 & $\mathrm{X}$ & 1.00 \\
\hline 3_Grade Subbase(h) & & 0.00 & & 0.00 \\
\hline 4_Install Drainage(a) & $\mathrm{X}$ & 1.00 & & 0.00 \\
\hline 4_Install Drainage (b) & $\mathrm{X}$ & 1.00 & & 0.00 \\
\hline 4_Install Drainage(c) & & 0.00 & & 0.00 \\
\hline 4_Install Drainage(d) & & 0.00 & $\mathrm{X}$ & 1.00 \\
\hline 4_Install Drainage(e) & & 0.00 & & 0.00 \\
\hline 4_Install Drainage(f) & & 0.00 & & 0.00 \\
\hline 5_Place OGDC Mainline(a) & $\mathrm{X}$ & 1.00 & & 0.00 \\
\hline 5_Place OGDC Mainline(b) & $\mathrm{X}$ & 1.00 & $\mathrm{X}$ & 1.00 \\
\hline 5_Place OGDC Mainline(c) & & 0.00 & & 0.00 \\
\hline 5_Place OGDC Mainline(d) & & 0.00 & & 0.00 \\
\hline 6_Pave E.B. Mainline(a) & $\mathrm{X}$ & 1.00 & $\mathrm{X}$ & 1.00 \\
\hline 6_Pave E.B. Mainline(b) & $\mathrm{X}$ & 1.00 & $\mathrm{X}$ & 1.00 \\
\hline 6_Pave E.B. Mainline(c) & & 0.00 & $\mathrm{X}$ & 0.87 \\
\hline 7_Place OGDC Ramps and Gaps(a) & $\mathrm{X}$ & 1.00 & $\mathrm{X}$ & 0.85 \\
\hline 7_Place OGDC Ramps and Gaps(b) & $\mathrm{X}$ & 1.00 & $\mathrm{X}$ & 1.00 \\
\hline 8_Pave E.B. Gaps and Ramps(a) & $X$ & 1.00 & $\mathrm{X}$ & 1.00 \\
\hline 8_Pave E.B. Gaps and Ramps(b) & $\mathrm{X}$ & 1.00 & $\mathrm{X}$ & 1.00 \\
\hline 8_Pave E.B. Gaps and Ramps(c) & $\mathrm{X}$ & 1.00 & $\mathrm{X}$ & 1.00 \\
\hline 9_Place Gravel Shoulder(a) & & 0.00 & & 0.00 \\
\hline 9_Place Gravel Shoulder(b) & & 0.00 & & 0.00 \\
\hline 9_Place Gravel Shoulder(c) & & 0.00 & & 0.00 \\
\hline 10_Slope Grading and Restoration E.B. (a) & $\mathrm{X}$ & 1.00 & $\mathrm{X}$ & 1.00 \\
\hline 10_Slope Grading and Restoration E.B. (b) & $\mathrm{X}$ & 1.00 & $\mathrm{X}$ & 1.00 \\
\hline 11_Stripe to Open Pavement E.B. & & 0.00 & & 0.00 \\
\hline 12_Relocate Barrier Wall & & 0.00 & & 0.00 \\
\hline 13_Re-stripe W.B. & & 0.00 & & 0.00 \\
\hline 14_All Lanes Open & $\mathrm{X}$ & 1.00 & $\mathrm{X}$ & 1.00 \\
\hline
\end{tabular}

tion management strategies.

\section{ACKNOWLEDGMENTS:}

This work was supported by the NSF grant SES0624118 to Amlan Mukherjee. Any opinions, findings and conclusions or recommendations expressed in this material are those of the authors and do not necessarily reflect views of the National Science Foundation.

\section{REFERENCES}

Anderson, G. R., A. Mukherjee, and N. Onder. 2009. "Traversing and querying constraint driven temporal networks to estimate construction contingencies.". Automation in Construction 18 (6): 798-813.

Battikha, M. G. 2008, APR. "Reasoning mechanism for construction nonconformance root-cause analysis". Journal of Construction Engineering and Management 134 (4): 280-288. 
Caldas, C., and L. Soibelman. 2003, JUL. "Automating hierarchical document classification for construction management information systems". Automation in Construction 12 (4): 395-406.

Hegazy, T., and W. Menesi. 2010, OCT. "Critical Path Segments Scheduling Technique". Journal of Construction Engineering and Management 136 (10): 1078-1085.

Kim, H., L. Soibelman, and F. Grobler. 2008, JUL. "Factor selection for delay analysis using Knowledge Discovery in Databases". Automation in Construction 17 (5): 550-560.

Lucko, G., and Z. W. Mitchell, Jr.. 2010, JAN. "Quantitative Research: Preparation of Incongruous Economic Data Sets for Archival Data Analysis". Journal of Construction Engineering and Management 136 (1): 49-57.

Rojas, E., and A. Mukherjee. 2005. "Interval Temporal Logic in General Purpose Situational Simulations". Journal of Computing in Civil Engineering 19 (1): 83-93.

Rojas, E., and A. Mukherjee. 2006. "A Multi-Agent Framework for General Purpose Situational Simulations in the Construction Management Domain". Journal of Computing in Civil Engineering 20 (6): 1-12.

Soibelman, L., and H. Kim. 2002, JAN. "Data preparation process for construction knowledge generation through knowledge discovery in databases". Journal of Computing in Civil Engineering 16 (1): 39-48.

Tang, P., D. Cass, and A. Mukherjee. 2013, SEPT. "Investigating the Effect of Construction Management Strategies on Project Greenhouse Gas Emissions". Journal of Cleaner Production 54:78-88.

Tang, P., A. Mukherjee, and N. Onder. 2010a, December. "Strategy optimization and generation for construction project management using an interactive simulation". In Proceedings of the 2010 Winter Simulation Conference, edited by B. Johansson, S. Jain, J. Montoya-Torres, J. Hugan, and E. Yücesan, 3088 -3099. Piscataway, New Jersey: Institute of Electrical and Electronics Engineers, Inc.

Tang, P., A. Mukherjee, and N. Onder. 2010b, May.. "Studying Dynamic Decision-Making in Construction Management Using Adaptive Interactive Simulations". In Construction Research Congress 2010: Innovation for Reshaping Construction Practice, Proceedings of the 2010 Construction Research Congress, 379-38.

\section{AUTHOR BIOGRAPHIES}

PEI TANG is a Project Controls Engineer in JCMS, Inc. He holds a PhD degree in Civil Engineering from Michigan Technological University. His professional interests are in project scheduling, risk analysis and management, construction simulation, and contract management. His web page can be found via www.husksnow.com.

AMLAN MUKHERJEE is an AssociateProfessor at the Department of Civil and Environmental Engineering at Michigan Technological University. His research interests are artificial intelligence technologies such as agent based modeling and temporal logic, construction engineering and management, interactive simulations, construction engineering education, and cognitive modeling of expert decision making. His web page can be found via http://www.cee.mtu.edu/ amlan

NILUFER ONDER is an Associate Professor at the Department of Computer Science at Michigan Technological University. Her research interests are artificial intelligence, planning, planning under uncertainty, and decision making under uncertainty. Her web page can be found via http://www.cs.mtu.edu/ nilufer 